\title{
Synthesis of Some New Pyrazolines via One-Pot Three Component Technique and Their Corresponding Thiazole Compounds
}

\author{
Rezan Huseen Hama Salih * and Farouq Emam Hawaiz ${ }^{* *}$ \\ *Department of Chemistry, College of Education, University of Garmian-Kalar, Kurdistan Region, Iraq. \\ *Department of Chemistry, College of Education, University of Salahaddin-Hawler, Erbil,Kurdistan Region, Iraq. \\ *rezan.huseen@yahoo.com or rezan.huseen@garmian.edu.krd
}

\begin{abstract}
In the present study; a one-pot three component reaction between4(4'-chlorobenzyloxy) acetophenone, thiosemicarbazide and different substituted benzaldehydes in absolute ethanol under reflux with stirring has been used to give a new series of pyrazoline derivatives \{3-(4-[4'chlorobenzyloxy)phenyl)-5-(substituted-phenyl]-4,5-dihydropyrazole-1-carbothioamide) in high yields and short reaction times. . The later compounds were converted to their corresponding thiazole derivatives through reacting with 4-bromophenacyl bromide in ethanol., The structures of the newly synthesized compounds were identified on the bases of their FT-IR, ${ }^{1} \mathrm{H}-\mathrm{NMR},{ }^{13} \mathrm{C}-$ NMR and DEPT-135 spectra.
\end{abstract}

Keywords: One-pot synthesis, acetophenone. Benzylation, Pyrazoline, Thiazole.

\section{INTRODUCTION}

A one -pot multicomponent reactions, are very important and attractive subjects in organic synthesis due to the combination of three or more components to synthesis a final product in just one vessel. [1]. The one step methods involving three-component condensation are popular in synthetic organic chemistry for the synthesis of heterocyclic compounds [2]. One -pot have inherent advantages over conventional methode in several aspects: reducing the number of synthetic steps, operational simplicity, require shorter reaction time and energy saving, low cost and high product yields[3].

Hetrocyclic compounds such as pyrazolines and thiazoles are attracted much considerable attention due to their wide spectrum of biological activiti which include: antibacterial [4], antiinflammatory [5] , antifungal [6] ,antioxidant[7],anti-depressant [8],anti-convulsant[9],anticancer[10], anti-amoebic[11], antimicrobial[12], anti-tuberc ular[13], antitumor[14]. 


\section{MATERIAL AND METHODS}

A mixture of 4-hydroxy-acetophenone (6.8 gm, $50 \mathrm{mmol})$, [4-chloro benzylchloride (9.66 gm, 60 mmol)] and anhydrous $\mathrm{K}_{2} \mathrm{CO}_{3}(13.82 \mathrm{gm}, 100 \mathrm{mmol})$, in ethanol (100 mL-99.9\%) was refluxed for (6) hrs. with stirring. When the reaction was completed by the changing of the color, the cooled solution poured into water, solid materials immediately was obtained. The desired product was filtered off, washed several times with water and cold ethanol, dried and recrystallized from ethanol to obtain white

\subsection{Experimental notes}

Melting points were determined using a Stuart Scientific melting point apparatus (SMP3). IR spectra were recorded on IR Affinity-1 Spectrophotometer, using $\mathrm{KBr}$ disc. ${ }^{1} \mathrm{H}-\mathrm{NMR},{ }^{13} \mathrm{C}$ NMR and ${ }^{13}$ C-DEPT-135 spectra were taken on a Bruker $400 \mathrm{MHz}$ ultra-shield with TMS as internal references, in Jordan University of science and Technology.

\subsection{Preparation of starting material 4-(4'-chlorobenzyloxy) acetophenone (1)}

crystal of 4-(4'-chloro benzyloxy)acetophenone(1)[15].

$\left(\mathrm{C}_{15} \mathrm{H}_{13} \mathrm{ClO}_{2}\right)$, m.p. $\left(91-93^{\circ} \mathrm{c}\right), \lambda_{\max }(336 \mathrm{~nm})$ with product yield of $(12.65 \mathrm{gm}, 97 \%)$; IR $\left(\mathrm{cm}^{-1}\right)$ str.,1668 (C=O), 1597 (C=C), 2945-2873 (- $\mathrm{CH}_{3} \quad$ \&-CH $\left.2_{2}\right) . \quad{ }^{1} \mathrm{H}-\mathrm{NMR}: \quad 2.57\left(\mathrm{~s}, \quad 3 \mathrm{H}, \mathrm{C}_{11}\right)$, 5.09(s,2H, $\left.\mathrm{C}_{5}\right), 6.99 \quad\left(\mathrm{~d}, 2 \mathrm{H}, \mathrm{C}_{7,7^{\prime}}\right), 7.35\left(\mathrm{~s}, 4 \mathrm{H}, \mathrm{C}_{3}, 3^{\prime}\right), \quad 793\left(\mathrm{~d}, 2 \mathrm{H}, \mathrm{C}_{2,2^{\prime}, 8,8^{\prime}}\right), \quad ;{ }^{13} \mathrm{C}-$ NMR:26.36: $\mathrm{C}_{11}, 69.32: \mathrm{C}_{5}, 114.51: \mathrm{C}_{7,7^{\prime}}, 128.78: \quad \mathrm{C}_{3,3^{\prime}}, 128.88: \mathrm{C}_{2,2^{\prime}}, 130.63: \mathrm{C}_{8,8^{\prime}}, 130.71$ : $\mathrm{C}_{4}{ }^{\prime}, 134.07: \mathrm{C} 9,134.69: \mathrm{C}_{1}, 162.32: \mathrm{C}_{6}, 196.7: \mathrm{C}_{10},{ }^{13} \mathrm{C}-\mathrm{DEPT}-135: 26.37: \mathrm{C}_{11},:-69.32: \mathrm{C}_{5}$, 114.51: $\mathrm{C}_{7,7^{\prime}}, 128.78: \mathrm{C}_{3,3}, 128.89: \mathrm{C}_{2,2^{\prime}}, 130.64: \mathrm{C}_{8,8^{\prime}}$

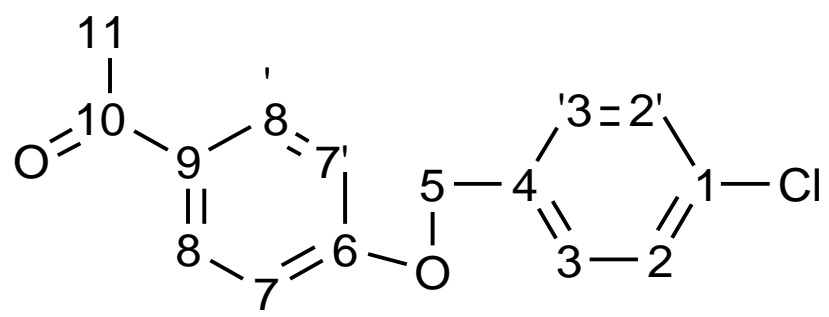

\section{3 one pot Synthesis of Pyrazolines: 3-(4-[4'-chlorobenzyloxy)phenyl)-5- (substituted-phenyl]-4,5-dihydropyrazole-1-carbothioamide(2a-e)}

A mixture of 4-(4-chlorobenzyloxy) acetophenone (1) (7 mmol, $1.8 \mathrm{gm})$, substituted benzaldehyde $(7 \mathrm{mmol}), \mathrm{NaOH}(4 \%, 12 \mathrm{mmol})$ and thiosemicarbazide $(10 \mathrm{mmol}, 0.9 \mathrm{gm})$ was 
mixed together in $30 \mathrm{~mL}$ ethanol $99.9 \%$ and refluxed with stirring for appropriate time until completion the reaction which was monitored by FT-IR and testing drops of mixture with conc. sulfuric acid which produced a green color. The solution was cooled and the solid product was separated, washed several times with cold ethanol dried and recrystallized from toluene to give pyrazolines ( 2 a-e)

\section{3a3(4-(4'-chlorobenzyloxy)phenyl)-5-(4-chlorophenyl)-4,5-dihydropyrazole- 1-carbothioamide.}

$\left(\mathrm{C}_{23} \mathrm{H}_{19} \mathrm{Cl}_{2} \mathrm{~N}_{3} \mathrm{O}_{1} \mathrm{~S}\right)$, m.p. (172-174), yield (87.6\%); IR ( $\left.\mathrm{cm}^{-1}\right)$ str., 3427,3278 $\left(\mathrm{NH}_{2)}, 1606(\mathrm{C}=\mathrm{C})\right.$, 1462(C=N), 1367(C=S). $\quad{ }^{1} \mathrm{H}-\mathrm{NMR}: \quad 3.12\left(\mathrm{dd}, \quad 1 \mathrm{H}, \quad-\mathrm{CH}_{2}-\mathrm{Ha}-\mathrm{C}_{11}\right), \quad 3.77\left(\mathrm{dd}, 1 \mathrm{H},-\mathrm{CH}_{2}-\mathrm{H}_{\mathrm{b}^{-}}\right.$ $\left.\mathrm{C}_{11}\right), 5.06\left(\mathrm{~s}, 2 \mathrm{H},-\mathrm{O}-\mathrm{CH}_{2}-\mathrm{C}_{5}\right), 5.97\left(\mathrm{dd}, 1 \mathrm{H},-\mathrm{CH}-\mathrm{H}_{\mathrm{X}}-\mathrm{C}_{12}\right), 6.44\left(\mathrm{~b} . \mathrm{s}, 2 \mathrm{H},-\mathrm{NH}_{2}\right), 6.79-8.88(\mathrm{~m}, 12 \mathrm{H}, \mathrm{Ar}-\mathrm{H}-$

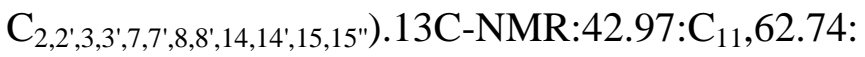

$\mathrm{C}_{12}, 69.29: \mathrm{C}_{5}, 114.81: \mathrm{C}_{7,7}, 115.18: \mathrm{C}_{15,15^{\prime}}, 123.37: \mathrm{C}_{9}, 126.95: \mathrm{C}_{14} \quad, 14^{\prime}, 127.9: \mathrm{C}_{3,3^{\prime}}, 128.19:$ $\mathrm{C}_{2,2^{\prime}}, 128.17: \mathrm{C}_{8,8^{\prime}}, 129: \mathrm{C}_{16}, 133.29: \mathrm{C}_{1}, 134.69: \mathrm{C}_{4}, 140.35: \mathrm{C}_{13}, 155$ 51: $\mathrm{C}_{10}, 160.72: \mathrm{C}_{6}, 176.33: \mathrm{C}_{17 .}{ }^{13} \mathrm{C}-$ DEPT-135:-42.79: $\mathrm{C}_{11}, 62.55: \mathrm{C}_{12},-69.07: \mathrm{C}_{5}, 114.62$ : $\mathrm{C}_{7,7}, 114.99: \mathrm{C}_{15,15^{\prime}}, 126$. $78: \mathrm{C}_{14,14^{\prime}}, 127.74: \mathrm{C}_{3,3^{\prime}}, 128.55: \mathrm{C}_{2,2^{\prime}}, 128.83: \mathrm{C}_{8,8^{\prime}}$

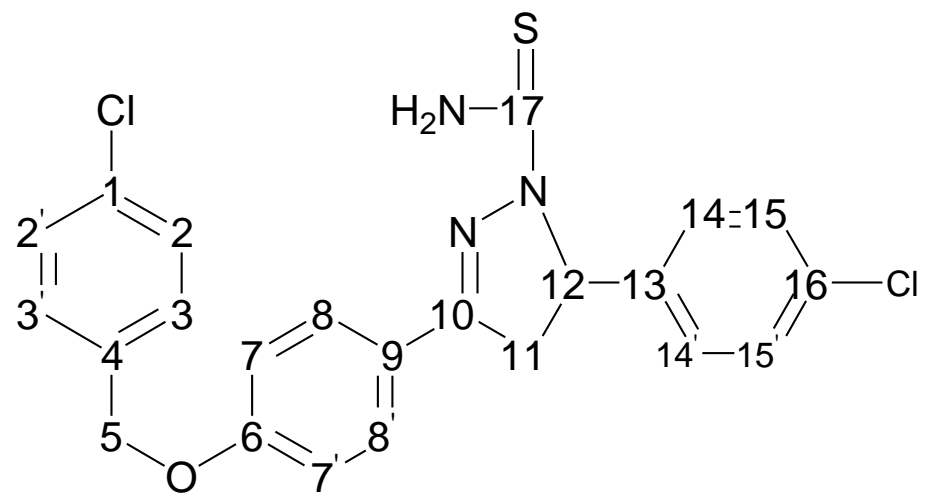

\section{3b 3(4-(4'-chlorobenzyloxy)phenyl)-5-(3-chlorophenyl)-4,5-dihydropyrazole- 1-carbothioamide}

$\left(\mathrm{C}_{23} \mathrm{H}_{19} \mathrm{Cl}_{2} \mathrm{~N}_{3} \mathrm{O}_{1} \mathrm{~S}\right)$, m.p. (198-199), yield (80.6\%); IR ( $\left.\mathrm{cm}^{-1}\right)$ str., $3402,3232\left(\mathrm{NH}_{2)}, 1589(\mathrm{C}=\mathrm{C})\right.$, $1498(\mathrm{C}=\mathrm{N}), 1375(\mathrm{C}=\mathrm{S})$.

\section{3c 3(4-(4'-chlorobenzyloxy)phenyl)-5-(4-fluorophenyl)-4,5-dihydropyrazole- 1-carbothioamide}

$\left(\mathrm{C}_{23} \mathrm{H}_{19} \mathrm{Cl}_{2} \mathrm{~N}_{3} \mathrm{O}_{1} \mathrm{~S}\right)$, m.p. (188-189), yield (78.7\%); IR (cm $\left.{ }^{-1}\right)$ str., 3475,3350 $\left(\mathrm{NH}_{2)}, 1608\right.$ $(\mathrm{C}=\mathrm{C}), 1475(\mathrm{C}=\mathrm{N}), 1361(\mathrm{C}=\mathrm{S}) .{ }^{1} \mathrm{H}-\mathrm{NMR}: 3.16\left(\mathrm{dd}, 1 \mathrm{H},-\mathrm{CH}_{2}-\mathrm{Ha}-\mathrm{C}_{11}\right), 3.79\left(\mathrm{dd}, 1 \mathrm{H},-\mathrm{CH}_{2}-\mathrm{H}_{b^{-}}\right.$ 
$\left.\mathrm{C}_{11}\right), 5.07\left(\mathrm{~s}, 2 \mathrm{H},-\mathrm{O}-\mathrm{CH}_{2}-\mathrm{C}_{5}\right), 5.98\left(\mathrm{dd}, 1 \mathrm{H},-\mathrm{CH}-\mathrm{H}_{\mathrm{X}}-\mathrm{C}_{12}\right), 6.21\left(\mathrm{~b} . \mathrm{s}, 2 \mathrm{H},-\mathrm{NH}_{2}\right), 6.55-7.66(\mathrm{~m}, 12 \mathrm{H}, \mathrm{Ar}-\mathrm{H}-$

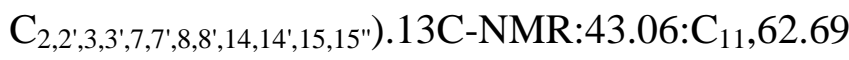

$: \mathrm{C}_{12}, 69.31: \mathrm{C}_{5}, 114.82: \mathrm{C}_{7,7^{\prime}}, 115.60: \mathrm{C}_{15,15^{\prime}}, 123.44: \mathrm{C}_{9}, 127.31: \mathrm{C}_{14,14^{\prime}}, 127.91: \mathrm{C}_{3,3^{\prime}}, 128.71: \mathrm{C}_{2,2^{\prime}}, 128.84:$

$\mathrm{C}_{8,8^{\prime}}, 134.71: \mathrm{C}_{1}, 134.96: \mathrm{C}_{13}, 137.64: \mathrm{C}_{4}, 155.56: \mathrm{C}_{10}, 160.77: \mathrm{C}_{16}, 163.26: \mathrm{C}_{6}, 176.31: \mathrm{C}_{17}{ }^{13} \mathrm{C}-\mathrm{DEPT}-$ 135:42.89: $\mathrm{C}_{11}, 62.47: \mathrm{C}_{12}, 69.11: \mathrm{C}_{5}, 114.61: \mathrm{C}_{7,7}, 115.43: \mathrm{C}_{15,15^{\prime}}, 127.12: \mathrm{C}_{14,14}, 127.74: \mathrm{C}_{3,3}, 128.56$ :

$\mathrm{C}_{2,2^{\prime}}, 128.67: \mathrm{C}_{8,8^{\prime}}$.

\section{3d3(4-(4'-chlorobenzyloxy)phenyl)-4,5-dihydro-5-(3-nitrophenyl)pyrazole-1- carbothioamide.}

$\left(\mathrm{C}_{23} \mathrm{H}_{19} \mathrm{Cl}_{2} \mathrm{~N}_{4} \mathrm{O}_{3} \mathrm{~S}\right.$ ),m.p. (166-168), yield (65.6\%); IR ( $\left.\mathrm{cm}^{-1}\right)$ str., 3433,3392 $\left(\mathrm{NH}_{2)}, 1602\right.$ $(\mathrm{C}=\mathrm{C}), 1462(\mathrm{C}=\mathrm{N}), 1338(\mathrm{C}=\mathrm{S})$.

\section{3e3(4-(4'-chlorobenzyloxy)phenyl)-4,5-dihydro-5-(4-nitrophenyl)pyrazole-1- carbothioamide.}

$\left(\mathrm{C}_{23} \mathrm{H}_{19} \mathrm{Cl}_{2} \mathrm{~N}_{4} \mathrm{O}_{3} \mathrm{~S}\right.$ ),m.p. (204-206), yield (71.9\%); IR ( $\left.\mathrm{cm}^{-1}\right)$ str., 3477,3344 ( $\mathrm{NH}_{2)}, 1606$ $(\mathrm{C}=\mathrm{C}), 1475(\mathrm{C}=\mathrm{N}), 1344(\mathrm{C}=\mathrm{S})$.

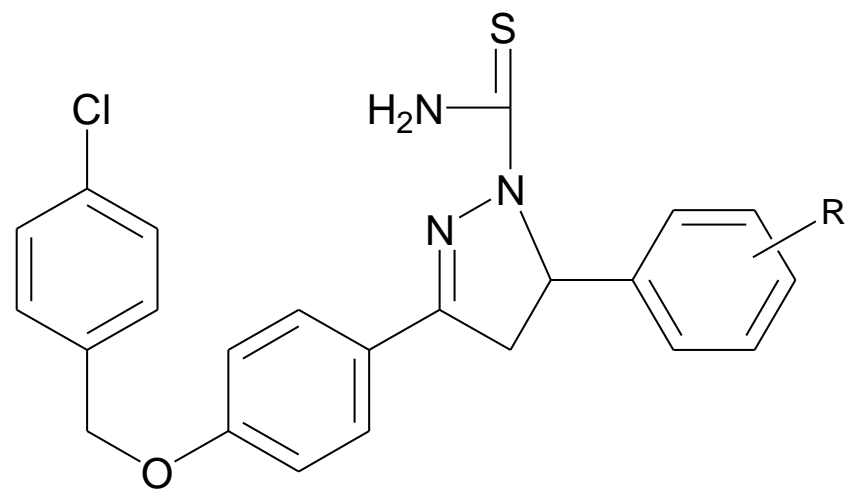

$\mathrm{R}=4-\mathrm{Cl}$, 3-Cl. 4- $\mathrm{F}, 3-\mathrm{NO}_{2}, 4-\mathrm{NO}_{2}$

\subsection{Synthesis of thiazoles: 3-(4-(4-chlorobenzyloxy)phenyl)-5-(substituted- phenyl)-1-(4-(4-bromophenyl)thiazol-2-yl)-4,5-dihydro-1H-pyrazole (3 a-e)}

A mixture of pyrazoline derivatives (3a-e) $(1 \mathrm{mmol})$, 4-bromophenacyl bromide (0.83gm, $3 \mathrm{mmol})$ and absolute ethanol (10 mL, 99.9\%) was refluxed with stirring for (1-4 hrs.) until completion the reaction which was monitored by both the changing of the color and the 
formation of ppt. The ppt was isolated by suction filtration, washed with ethanol, dried and purified by recrystallization from toluene and ethanol as a suitable solvent.

\section{4a 3-(4-(4'-chlorobenzyloxy)phenyl)-1-(4-(4-bromophenyl)thiazole-2-yl)-5-(4- chlorophenyl)-4,5-dihydro-1H-pyrazole.}

$\left(\mathrm{C}_{31} \mathrm{H}_{22} \mathrm{BrCl}_{2} \mathrm{~N}_{3} \mathrm{O}_{1} \mathrm{~S}\right)$, m.p. (193-194), yield (98\%); IR $\left(\mathrm{cm}^{-1}\right)$ str., $1602(\mathrm{C}=\mathrm{C}), 1541(\mathrm{C}=\mathrm{N})$. ${ }^{1} \mathrm{H}-\mathrm{NMR}: \quad 3.26\left(\mathrm{dd}, \quad 1 \mathrm{H}, \quad-\mathrm{CH}_{2}-\mathrm{Ha}-\mathrm{C}_{11}\right), \quad 3.87\left(\mathrm{dd}, \quad 1 \mathrm{H}, \quad-\mathrm{CH}_{2}-\mathrm{H}_{\mathrm{b}}-\mathrm{C}_{11}\right), 5.07 \quad\left(\mathrm{~s}, 2 \mathrm{H},-\mathrm{O}-\mathrm{CH}_{2}-\right.$ $\left.\mathrm{C}_{5}\right), 5.57\left(\mathrm{dd}, 1 \mathrm{H},-\mathrm{CH}-\mathrm{H}_{\mathrm{X}}-\mathrm{C}_{12}\right), 6.80\left(. \mathrm{s}, 1 \mathrm{H},-\mathrm{CH}-\mathrm{C}_{19}\right), 6.92-7.76(\mathrm{~m}, 16 \mathrm{H}$, $\mathrm{Ar}-\mathrm{H}-$

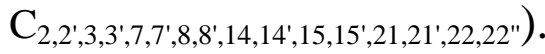

\section{4b3-(4-(4'-chlorobenzyloxy)phenyl)-1-(4-(4-bromophenyl)thiazole-2-yl)-5-(3- chlorophenyl)-4,5-dihydro-1H-pyrazole.}

$\left(\mathrm{C}_{31} \mathrm{H}_{22} \mathrm{BrCl}_{2} \mathrm{~N}_{3} \mathrm{O}_{1} \mathrm{~S}\right)$, m.p. (205-207), yield (96.8\%); IR ( $\left.\mathrm{cm}^{-1}\right)$ str., $1591(\mathrm{C}=\mathrm{C}), 1512(\mathrm{C}=\mathrm{N})$.

\section{4c3-(4-(4'-chlorobenzyloxy)phenyl)-1-(4-(4-bromophenyl)thiazole-2-yl)-5-(4- fluorophenyl)-4,5-dihydro-1H-pyrazole.}

$\left(\mathrm{C}_{31} \mathrm{H}_{22} \mathrm{BrCl}_{1} \mathrm{~N}_{3} \mathrm{O}_{1} \mathrm{FS}\right)$, m.p. (216-217), yield (71\%); IR $\left(\mathrm{cm}^{-1}\right)$ str., $1604(\mathrm{C}=\mathrm{C}), 1546(\mathrm{C}=\mathrm{N}) .{ }^{1} \mathrm{H}-$ NMR: $\quad 3.28\left(\mathrm{dd}, \quad 1 \mathrm{H}, \quad-\mathrm{CH}_{2}-\mathrm{Ha}-\mathrm{C}_{11}\right), \quad 3.87\left(\mathrm{dd}, \quad 1 \mathrm{H}, \quad-\mathrm{CH}_{2}-\mathrm{H}_{\mathrm{b}}-\mathrm{C}_{11}\right), 5.08 \quad\left(\mathrm{~s}, 2 \mathrm{H},-\mathrm{O}-\mathrm{CH}_{2}-\right.$ $\left.\mathrm{C}_{5}\right), 5.59\left(\mathrm{dd}, 1 \mathrm{H},-\mathrm{CH}-\mathrm{H}_{\mathrm{X}}-\mathrm{C}_{12}\right), 6.80\left(. \mathrm{s}, 1 \mathrm{H},-\mathrm{CH}-\mathrm{C}_{19}\right), 6.98-7.71(\mathrm{~m}, 16 \mathrm{H}, \mathrm{Ar}-\mathrm{H}-$

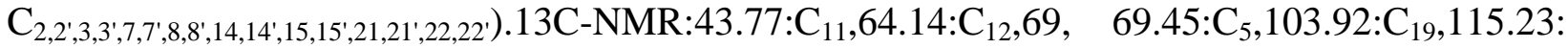
$\mathrm{C}_{7,7^{\prime}}, 115.81: \mathrm{C}_{15,15^{\prime}}, 121.41: \mathrm{C}_{23}, 124.59: \mathrm{C}_{9}, 127.53: \mathrm{C}_{14,14^{\prime}}, 128.16: \mathrm{C}_{3,3^{\prime}}, 128.43: \mathrm{C}_{2,2^{\prime}}, 128.51: \mathrm{C}_{21,21}, 128$. 91: $\mathrm{C}_{8,8}, 129: \mathrm{C}_{20}, 131.64: \mathrm{C}_{22,22}, 134.13: \mathrm{C}_{1}, 135.13: \mathrm{C}_{13}, 137.72: \mathrm{C}_{4}, 150.54: \mathrm{C}_{18}, 151.6: \mathrm{C}_{10}, 160.06: \mathrm{C}_{16}, 1$ 61.15: $\mathrm{C}_{6}, 165.30: \mathrm{C}_{17 \cdot}{ }^{13} \mathrm{C}-\mathrm{DEPT}-135:-43.58: \mathrm{C}_{11}, 63.93: \mathrm{C}_{12},-69.25: \mathrm{C}_{5}, 103.72: \mathrm{C}_{19}, 115.03:$

$\mathrm{C}_{7,7^{\prime}}, 115.62: \mathrm{C}_{15,15^{\prime}}, 127.34: \mathrm{C}_{14,14^{\prime}}, 127.97: \mathrm{C}_{3,3^{\prime}}, 128.23: \mathrm{C}_{2,2^{\prime}}, 128.31: \mathrm{C}_{21,21^{\prime}}, 128.71: \mathrm{C}_{8,8^{\prime}}, 131.45: \mathrm{C}_{22,22^{\prime}}$.

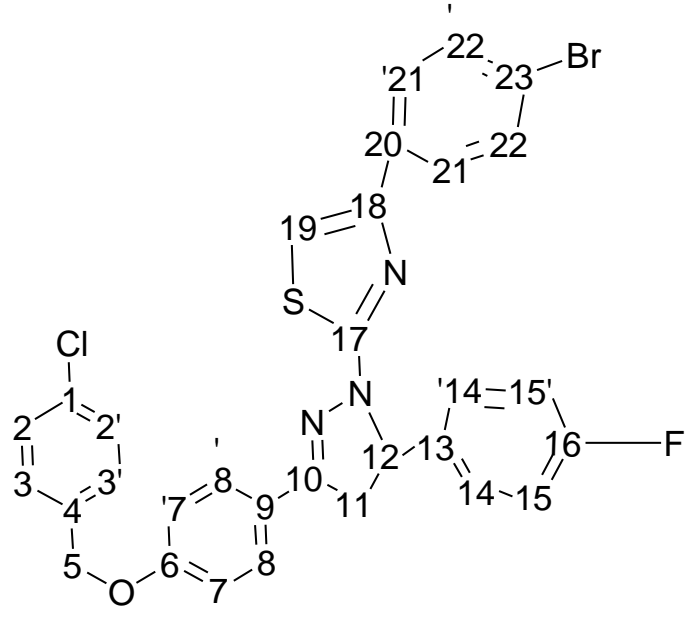


2.4d3-(4-(4'-chlorobenzyloxy)phenyl)-1-(4-(4-bromophenyl)thiazole-2-yl)-4,5dihydro-5-(3-nitrophenyl) -1H-pyrazole.

$\left(\mathrm{C}_{31} \mathrm{H}_{22} \mathrm{BrCl}_{1} \mathrm{~N}_{4} \mathrm{O}_{3} \mathrm{~S}\right)$, m.p. (131-133), yield (71\%); IR $\left(\mathrm{cm}^{-1}\right)$ str., $1604(\mathrm{C}=\mathrm{C}), 1525(\mathrm{C}=\mathrm{N})$.

2.5e3-(4-(4'-chlorobenzyloxy)phenyl)-1-(4-(4-bromophenyl)thiazole-2-yl)-4,5dihydro-5-(4-nitrophenyl) -1H-pyrazole.

$\left(\mathrm{C}_{31} \mathrm{H}_{22} \mathrm{BrCl}_{1} \mathrm{~N}_{4} \mathrm{O}_{3} \mathrm{~S}\right)$, m.p. (115-117), yield (79\%); IR $\left(\mathrm{cm}^{-1}\right)$ str., $1602(\mathrm{C}=\mathrm{C}), 1539(\mathrm{C}=\mathrm{N})$.

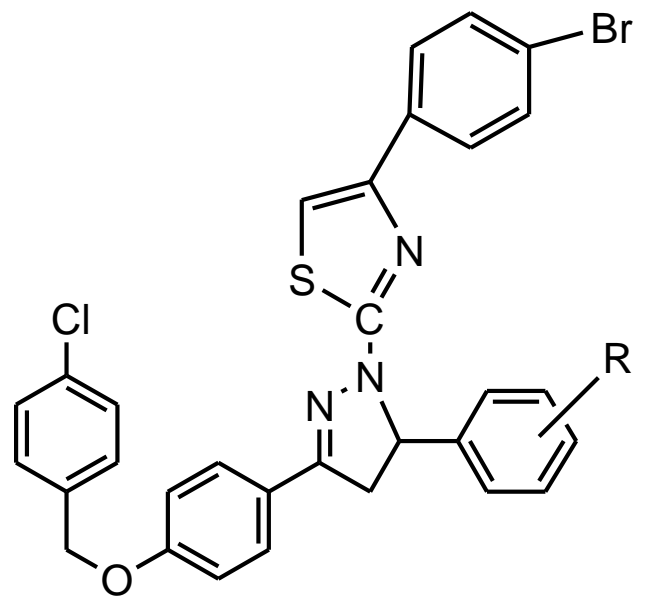

$\mathrm{R}=4-\mathrm{Cl}, 3-\mathrm{Cl} .4-\mathrm{F}, 3-\mathrm{NO}_{2}, 4-\mathrm{NO}_{2}$

\section{RESULTS AND DISCUSSION}

\subsection{Synthesis and characterization}

In a continuation of our attention on the synthesis of new pyrazolines using one-pot three component method, herein we utilized different substituted benzaldehydes in order to give new pyrazoline derivatives in high yields, reducing the number of synthetic steps, operational simplicity, shorter reaction time and energy saving via one -pot synthesis and their transformation to corresponding thiazole compounds Scheme (1). The IR spectra of compound (2 a-e)(Fig1) showed prominent two peaks at (3400-3200) $\mathrm{cm}^{-1}$ to the $\left(\mathrm{NH}_{2}\right)$ stretching of carbothioamide group attached to pyrazoline ring and disappearance of carbonyl group for both 
benzyloxy-acetophenone and substituted benzaldehyde. The ${ }^{1} \mathrm{H}-\mathrm{NMR}$ of compound (2 a) (Fig.2) showed characteristic three doublet to doublet (dd) signals corresponding to three protons (two geminal and one vicinal) of $\mathrm{C}_{11}$ and $\mathrm{C}_{12}$ of pyrazoline ring. . In ${ }^{13} \mathrm{C}-\mathrm{NMR}$ spectral data of compound ( 2 a) (Fig.3) there are three peaks in the high field region corresponding to $\left(\mathrm{C}_{11}, \mathrm{C}_{12}\right)$ of the pyrazoline ring and $\left(\mathrm{C}_{5}\right)$ of the benzyloxy group at $(42.97,62.74$ and 69.29$)$ respectively, also one singlet signal to $(\mathrm{C}=\mathrm{S})$ group were appeared at (176.33) ppm and the other peaks at (114.81-160.78) ppm for aromatic carbon atom. The ${ }^{13} \mathrm{C}$-DEPT-135 spectral information of compound (2 a) (Fig. 4 ) displayed two downward signals attributed to $\left(\mathrm{C}_{11}\right.$ and $\left.-\mathrm{O}-\mathrm{CH}_{2}-\mathrm{C}_{5}\right)$ at (42.79 and 69.07) consequently. The other upward signals corresponding to mono-protonated carbon atoms, and the disappearance signals of non-protonated carbon atoms in the aromatic ring $\left(\mathrm{C}_{1}, \mathrm{C}_{4}, \mathrm{C}_{6}, \mathrm{C}_{9}, \mathrm{C}_{10}, \mathrm{C}_{13}, \mathrm{C}_{16}\right.$, and $\left.\mathrm{C}_{17}\right)$ The good evidence to produce thiazole rings includes in the IR spectra of compounds (3a-e)(Fig5) the disappearance of $\left(\mathrm{NH}_{2}\right)$ stretching vibrations of carbothioamide group attached to pyrazoline ring at (3400-3200) $\mathrm{cm}^{-1}$ The ${ }^{1} \mathrm{H}-\mathrm{NMR}$ of thiazole compound (3 c) (Fig.6) display characteristic three doublet to doublet (dd) signals corresponding to three protons (two geminal and one vicinal) of $\mathrm{C}_{11}$ and $\mathrm{C}_{12}$ of pyrazoline ring and increase the signals of aromatic rings. ${ }^{13} \mathrm{C}-\mathrm{NMR}$ spectral data of thiazole (3 c) (Fig.7) exhibited three signals in the high field region corresponding to $\left(\mathrm{C}_{11}, \mathrm{C}_{12}\right.$ and $\left.\mathrm{C}_{5}\right)$ carbon atoms at (43.58, 63.93 and 69.25) consequently .In ${ }^{13}$ C-DEPT-135 spectral of compound (3 c) (Fig. 8) exhibited two downward signals at at (43.58 and 69.25) ppm fitted to the di-protonated $\left(\mathrm{C}_{11}\right.$ and $\mathrm{O}-\mathrm{CH}_{2}-\mathrm{C}_{5}$ ) carbon atoms, besides the appearance of other upward signals corresponding to mono-protonated carbon atoms. The disappearance signals of non-protonated carbon atoms in the aromatic region $\left(\mathrm{C}_{1}, \mathrm{C}_{4}, \mathrm{C}_{6}, \mathrm{C}_{9}, \mathrm{C}_{10}, \mathrm{C}_{13}, \mathrm{C}_{16}, \mathrm{C}_{17}, \mathrm{C}_{18}, \mathrm{C}_{20}\right.$ and $\left.\mathrm{C}_{23}\right)$. 

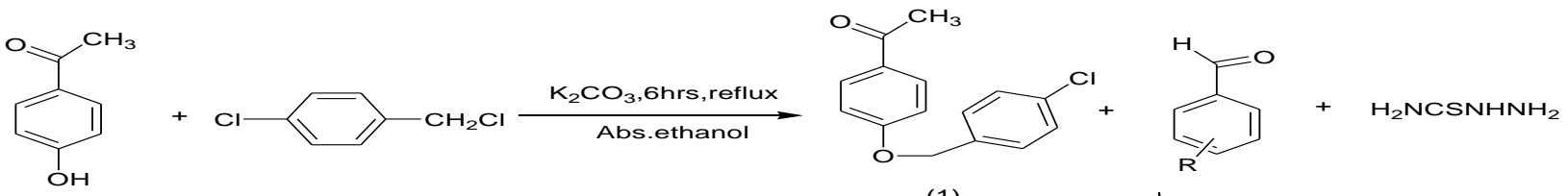

(1)

Via one pot -three component

NaOH, ethanol reflux(6)hrs
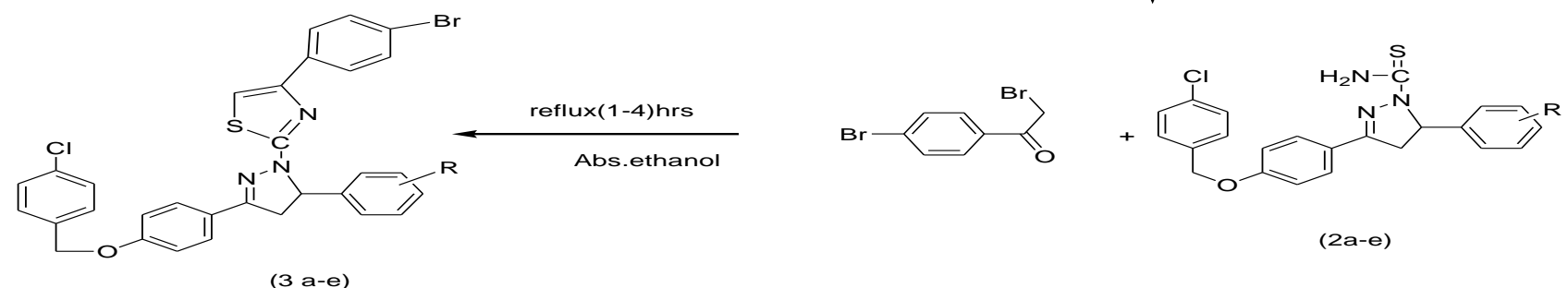

$\mathrm{R}=4-\mathrm{Cl}, 3-\mathrm{Cl}, 4-\mathrm{F}, 3-\mathrm{NO}_{2}, 4-\mathrm{NO}_{2}$.

Scheme 1

\section{CONCLUSION}

The goal of this work is to synthesis and spectroscopic characterization of some new substituted pyrazolines via one -pot-three component and their transformation to thiazole derivatives with a benzyloxy side chain.

In this study, we report a facile and efficient method for the synthesis of pyrazoline derivatives by three-component reaction between 4(4'-chlorobenzyloxy) acetophenone, substituted benzaldehyde and thiosemicarbazide. This method offers several advantages including reducing the number of synthetic steps, simple work-up procedure, require shorter reaction time and energy saving, less expensive and high product yields

\section{ACKNOWLEDGMENTS}

This study was supported by Chemistry Department, College of Education, Garmian University-Kalar and Salahaddin University-Hawler, Erbil, Kurdistan region, Iraq.

\section{References:}

1- HAJIPOUR, A.R., GHAYEB,Y., SHEIKHAN, N.\& RUOHO, A.E. (2009).Bronsted acidic ionic liquid as an efficient and reusable catalyst for one-pot synthesis of 1-amidoalkyl 2naphhols under solvent -free conditions. Tetrahedron Letters, (50), pp. 5649-5651. 
2- DEShMuKH, M. B., SAlunKhe, S. M., PATIL, D. R. \& ANBhule, P. V. (2009). A novel and efficient one step synthesis of 2-amino-5-cyano-6-hydroxy-4-aryl pyrimidines and their anti-bacterial activity. European journal of medicinal chemistry, 44(6), pp.26512654.

3- ABLAJAN, K., KAMIL, W., TUOHETI, A. \& WAN-FU, S. (2012). An efficient three component one-pot synthesis of 5-amino-7-aryl-7, 8-dihydro-[1, 2, 4] triazolo [4, 3-a]pyrimidine-6-carbonitriles. Molecules, 17(2), pp.1860-1869.

4- SOLANKEE, A., LAD, S., SOLANKEE, S. \& PATEL, G. (2009). Chalcones, pyrazolines and aminopyrimidines as antibacterial agents. Indian journal of chemistry, 48B(10), pp14421446.

5- GöKHAN-KELEKCI, N., YABANOĞLU, S., KUPELI, E., SAlGıN, U., ÖZGEN, Ö., UCAR, G., YEŞILADA, E., KENDI, E., YEŞILADA, A. \& BILGIN, A. A. (2007). A new therapeutic approach in Alzheimer disease: some novel pyrazole derivatives as dual MAO-B inhibitors and antiinflammatory analgesics. Bioorganic \& medicinal chemistry, 15(17), pp.5775-5786.

6- ALI, I., WANI, W. A., KHAN, A., HAQUE, A., AHMAD, A., SALEEM, K. \& MANZOOR, N. (2012). Synthesis and synergistic antifungal activities of a pyrazoline based ligand and its copper (II) and nickel (II) complexes with conventional antifungals. Microbial pathogenesis, 53(2), pp.66-73.

7- DASARY, K., LAVANIA, A., YADAV, M. \& ANAND, A. V. (2013). Synthesis, Characterization and Study of Antioxidant Activities of Some New Pyrazoline Derivatives Containing Isatin Moiety. International Journal of Research in Engineering and Science, 1(7), pp.8-13.

8- PALASKA, E., AYTEMIR, M., UZBAY, I. T. \& EROL, D. (2001). Synthesis and antidepressant activities of some 3, 5-diphenyl-2-pyrazolines. European journal of medicinal chemistry, 36(6), pp.539-543.

9- VALARMATHY, J., SAMUEL JOSHUA, L., SENTHIL KUMAR, K. L. \& KASABE, A. J. (2010). Synthesis, antitubercular, anticonvulsant and antiinflammatory activities of some novel 2-pyrazoline derivatives. Oriental Journal of Chemistry, 26(3), pp.1049-1054.

10- DAWOUD, N. T. (2014). Synthesis, Antimicrobial, Antifungal and Anticancer activities of Some New Heterocyclic Compounds incorporating Pyrazoline moiety. International Journal of Advanced Scientific and Technical Research, 3(4), pp.166-189. 
11- ABID, M. \& AZAM, A. (2006). Synthesis, characterization and antiamoebic activity of 1(thiazolo [4, 5-b] quinoxaline-2-yl)-3-phenyl-2-pyrazoline derivatives. Bioorganic \& medicinal chemistry letters, 16(10), pp. 2812-2816.

12- SOLANKEE, A. \& TAILOR, R. (2016). An efficient synthesis of some new chalcone, acetyl pyrazoline and amino pyrimidine bearing 1, 3, 5-triazine nucleus as potential antimicrobial and antitubercular agent. Chemistry International, 2(4), pp.189-200.

13- RANA, D. N., CHHABRIA, M. T., SHAH, N. K. \& BRAHMKSHATRIYA, P. S. (2014). Discovery of new antitubercular agents by combining pyrazoline and benzoxazole pharmacophores: design, synthesis and insights into the binding interactions. Medicinal Chemistry Research, 23(5), pp.2218-2228.

14- TAYLOR, E. C. \& PATEL, H. H. (1992). Synthesis of pyrazolo 3, 4-dpyrimidine analogues of the potent agent N-4-2-2-amino-4 3H-oxo-7H-pyrrolo 2, 3-dpyrimidin-5-yl ethylbenzoyl-L-glutamic acid (LY231514). Tetrahedron, 48(37), pp.8089-8100.

15- PLOURDE, G. L. \& SPAETZEL, R. R. (2002). Regioselective Protection of the 4-Hydroxyl of 3, 4-Dihydroxy-benzaldehyde. Molecules, 7(9), pp.697-705.

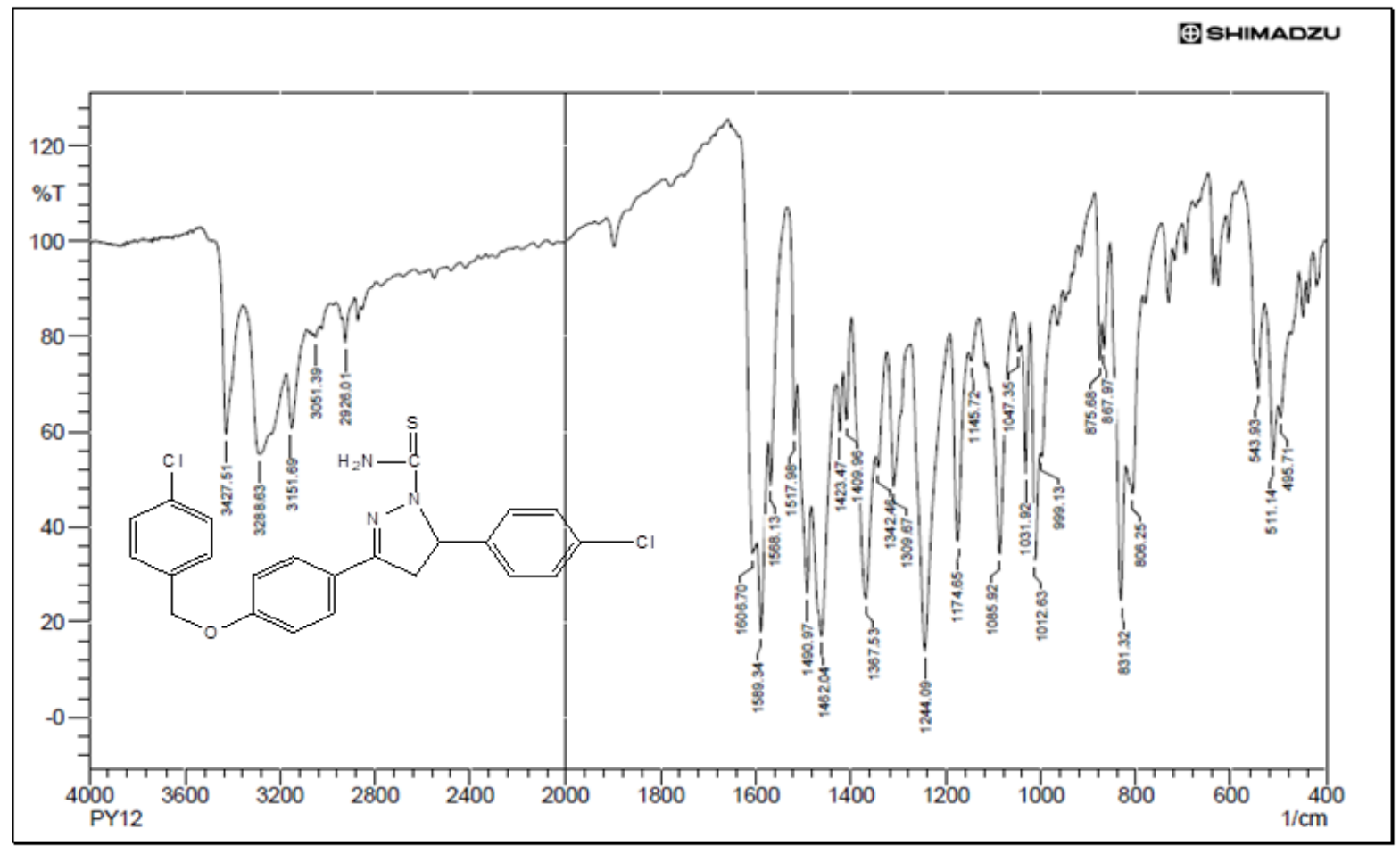

(Fig.1): FT-IR spectrum of compound. (2 a) 


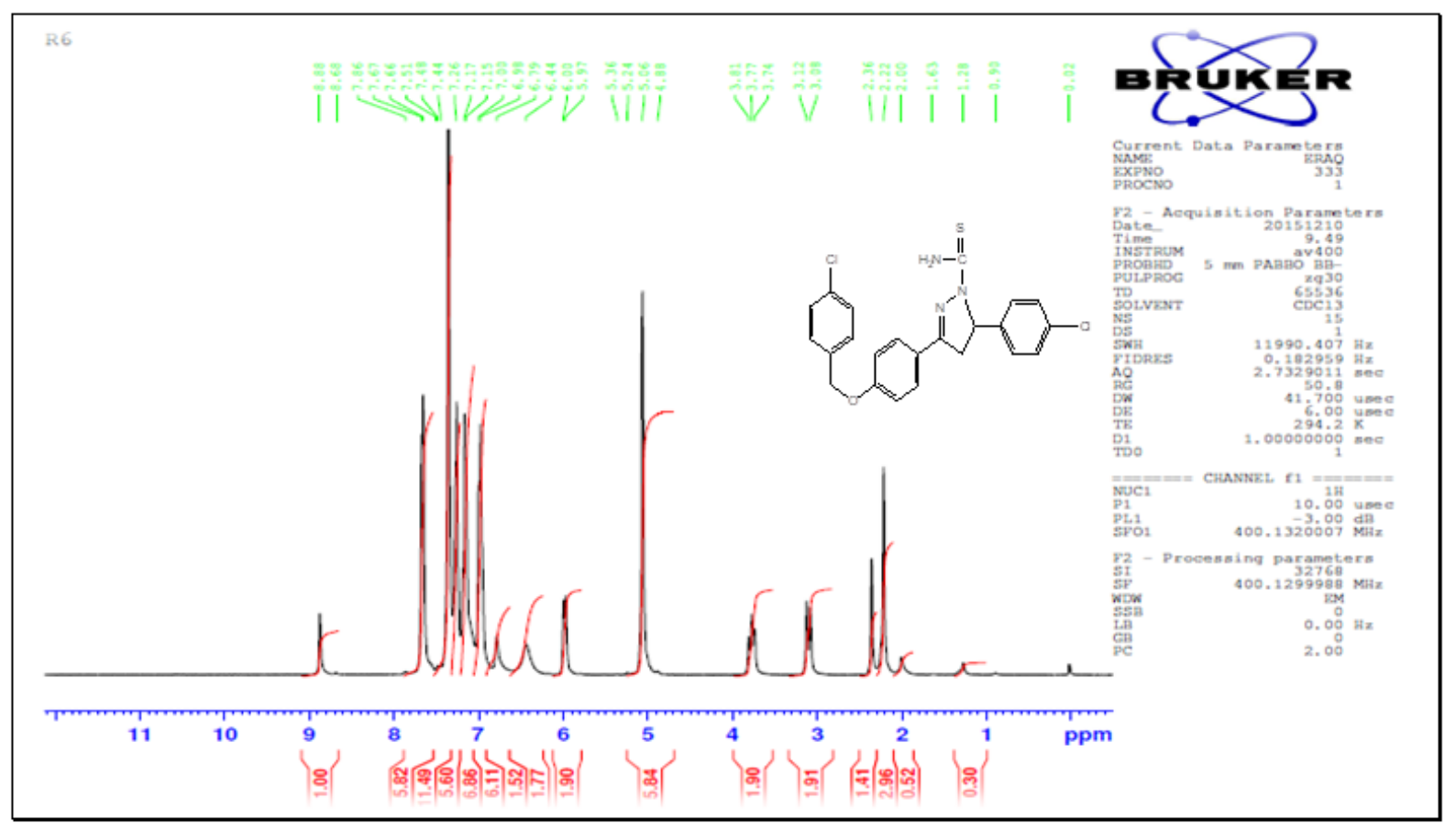

(Fig.2): ${ }^{1}$ H-NMR spectrum of compound. (2 a)

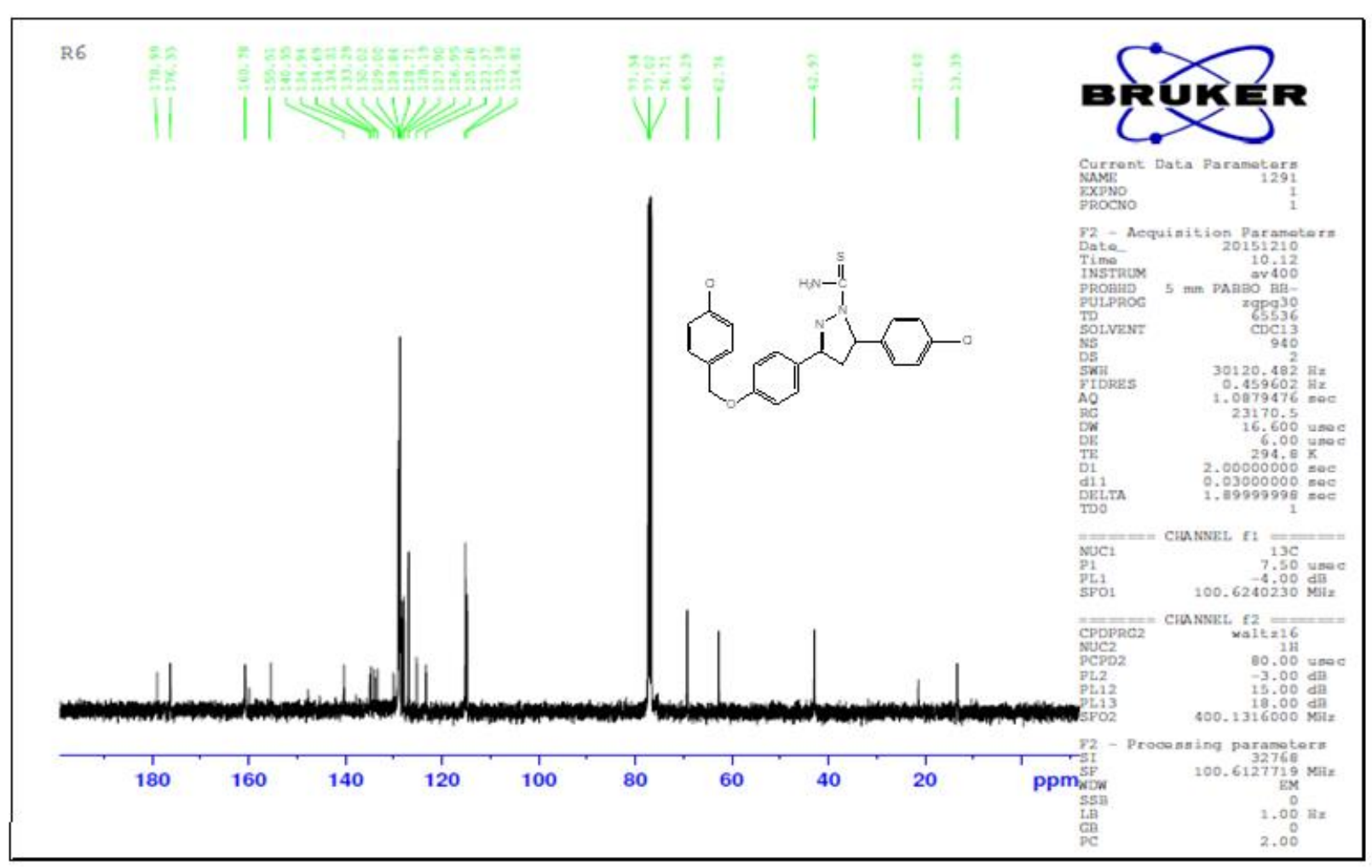

(Fig.3): ${ }^{13}$ C-NMR spectrum of compound. (2 a) 


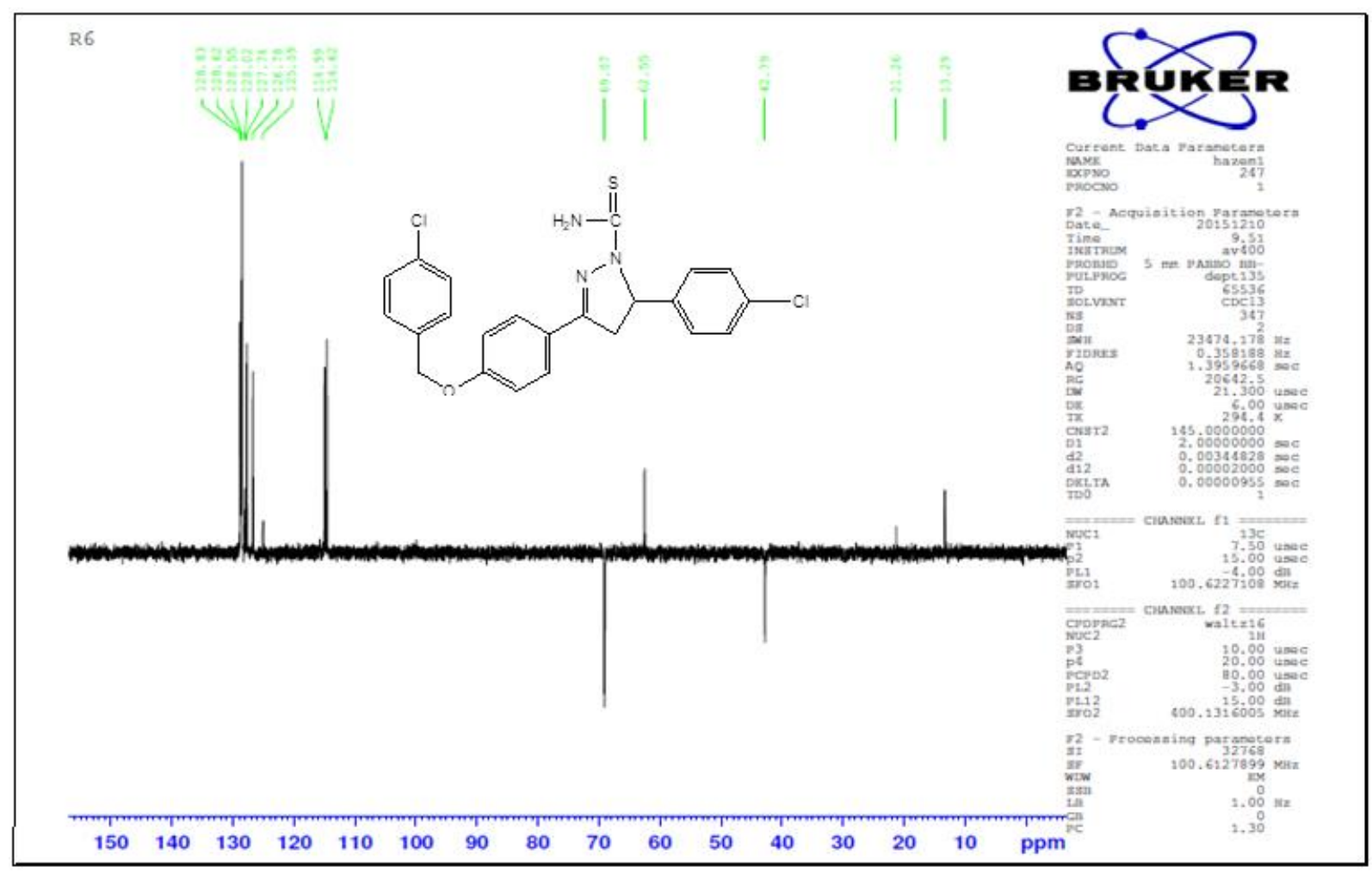

(Fig.4): ${ }^{13}$ C-DEPT NMR spectrum of compound. (2a)

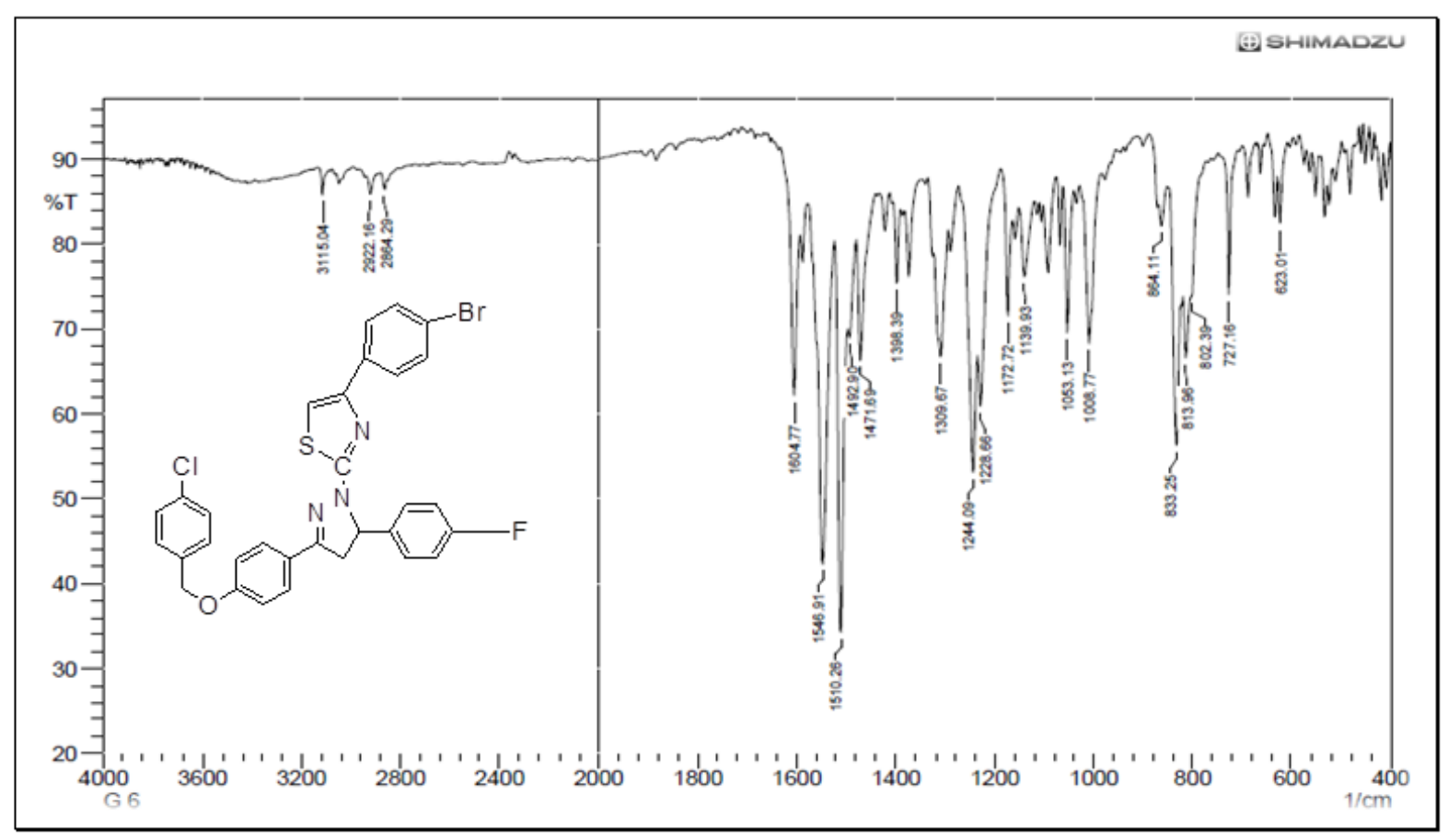

(Fig.5): FT-IR spectrum of compound. (3 c) 


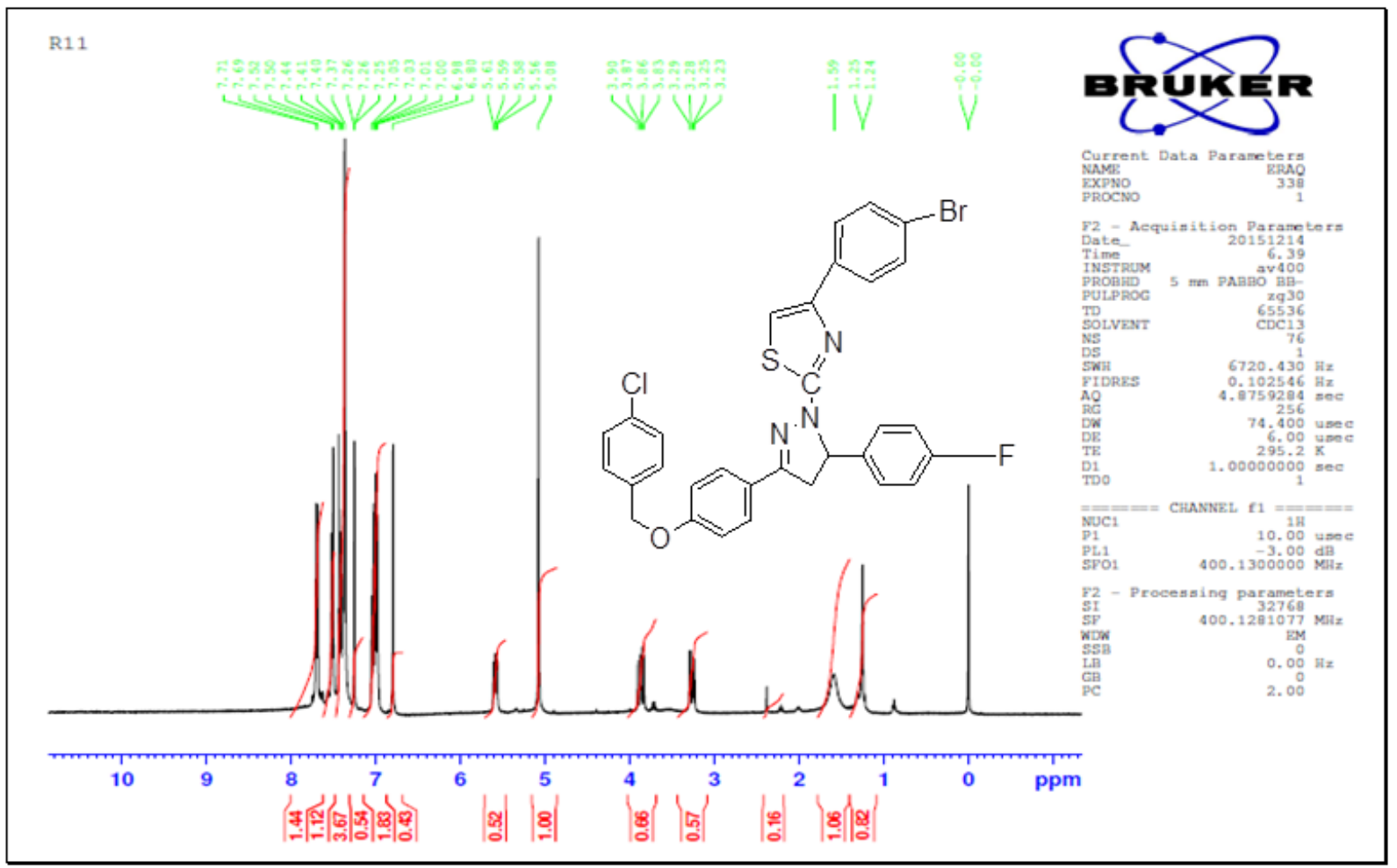

(Fig.6): ${ }^{1} \mathrm{H}-\mathrm{NMR}$ spectrum of compound. (3 c)

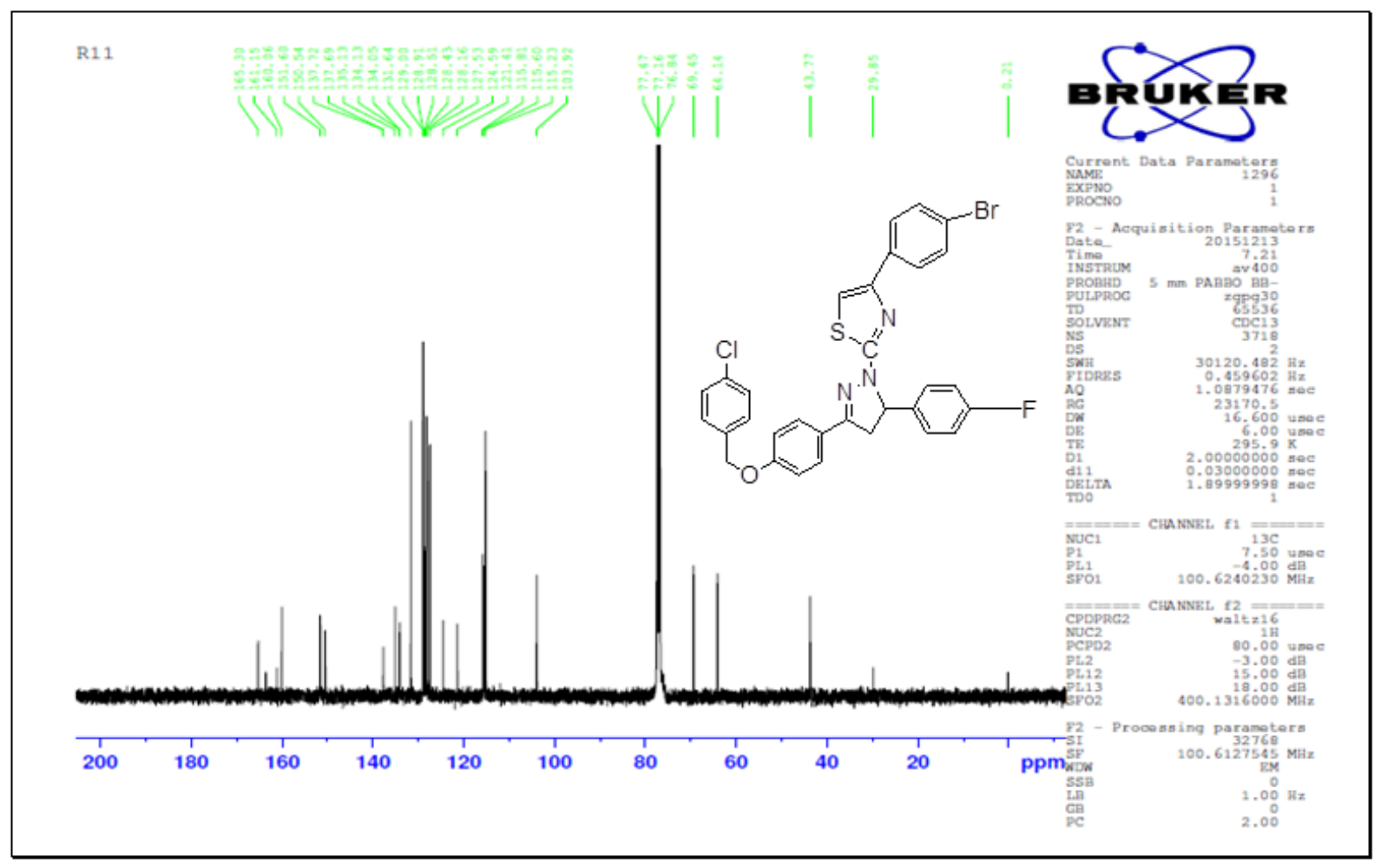

(Fig.7): ${ }^{13} \mathrm{C}-\mathrm{NMR}$ spectrum of compound. (3 c) 


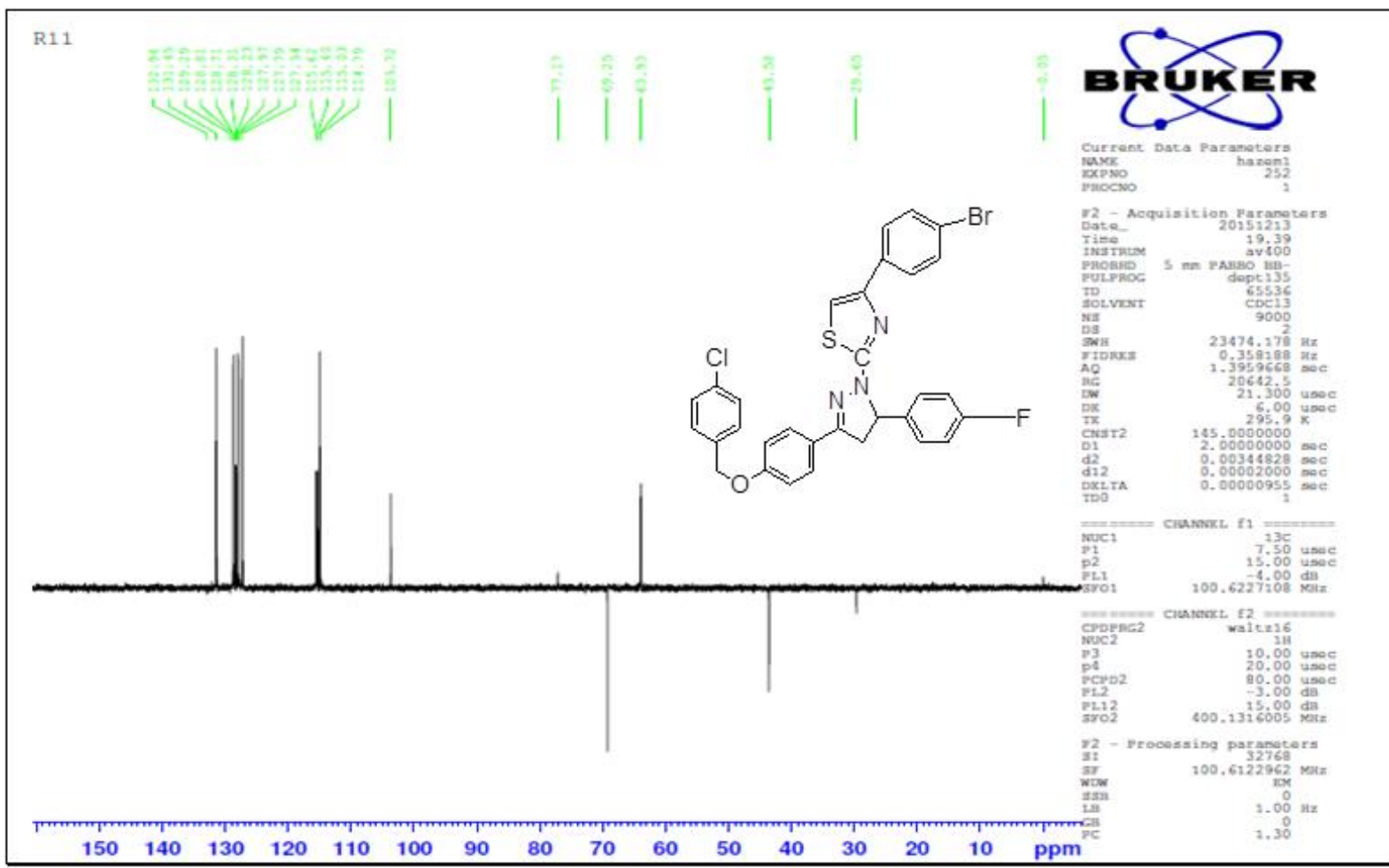

(Fig.8): ${ }^{13} \mathrm{C}$-DEPT-135 NMR spectrum of compound. (3 c) 